\title{
Measurement of Richtmyer-Meshkov mode coupling under steady shock conditions and at high energy density
}

\author{
C. A. Di Stefano*, C. C . Kuranz, S. R. Klein, R. P. Drake \\ Department of Atmospheric, Oceanic, and Space Sciences, University of Michigan, Ann Arbor, MI 48109, USA \\ G. Malamud* \\ Department of Atmospheric, Oceanic, and Space Sciences, University of Michigan, Ann Arbor, MI 48109, USA \\ Department of Physics, Nuclear Research Center - Negev, Beer-Sheva, Israel
}

\begin{abstract}
We present experiments observing Richtmyer-Meshkov mode coupling and bubble competition in a system arising from wellcharacterized initial conditions and driven by a strong (Mach 8) shock. These measurements and the analysis method developed to interpret them provide an important step towards the possibility of observing self-similarity under such conditions, as well as a general platform for performing and analyzing hydrodynamic instability experiments. A key feature of these experiments is that the shock is sustained sufficiently long that this nonlinear behavior occurs without decay of the shock velocity or other hydrodynamic properties of the system, which facilitates analysis and allows the results to be used in the study of analytic models.
\end{abstract}

\section{Introduction}

The growth of structure due to Richtmyer-Meshkov [1, 2] (RM) physics, occurring when a shock wave crosses an interface between two materials of differing densities, influences the subsequent dynamics and the interpenetration of the materials in many physical systems. Processes of interest range from the technological, such as the cooling of fuel pellets in inertial-confinement fusion [3], to many natural phenomena in which shocks play a role. The latter category includes various astrophysical events, such as jet dynamics [4], the mixing of layers in supernova remnants [5], and the dynamics of supernovae themselves, in which RM may also act as a seed for the faster-growing Rayleigh-Taylor (RT) instability [6, 7, 8, 9].

We recently reported the results of experiments [10] providing an exact measurement of a two-dimensional, two-mode RM interface in a high-energy-density (HED) system in which strong, unvarying shock conditions (Mach number $M \sim 8$ ) are sustained sufficiently long to observe nonlinear behavior of the system. This experiment produced the first observations of a purely RM system in which the dynamics were well-resolved over multiple time scales, including the linear phase as well as multiple later nonlinear phases. At these later times, the two initially-present perturbation modes first couple to produce new modes, ending the linear phase earlier than would be expected from any one of those individual modes in isolation, and subsequently enter into competition with each other, resulting

\footnotetext{
${ }^{*}$ Corresponding authors

Email addresses: carlosds@umich.edu (C. A. Di Stefano), gmalamud@umich.edu (G. Malamud)
}

in the reabsorption of higher-frequency modes into the lowerfrequency ones. This latest-time behavior is of particular interest, as theoretical models $[11,12]$ predict an eventual selfsimilar phase in which the rate of mode merging has converged to a characteristic value, although the exact prediction varies according to assumptions made about the physics.

The work presented here intends to lay the foundation for an analysis method that could lead to a direct measurement of this self-similar rate. We do this by first discussing the hydrodynamics of our two-mode experiment in detail, and then by describing a method for interpreting the data using Fourier analysis. This analysis method is important because the goal is to extend the experimental method to a similar system featuring much more complex dynamics, arising from an interface seeded with many modes [13]. However, in this case, a formal analysis method that does not rely on direct visual inspection is necessary, as the mode content of individual RM bubbles would no longer be easily discerned by sight. Such a multimode interface would allow for mode absorption to occur on many time scales simultaneously, offering the possibility of achieving selfsimilarity at late enough times.

Previous experiments have produced results consistent with such self-similarity for a three-dimensional system system [14], when considered alongside theoretical models accounting for three-dimensional effects [15]. These studies are highly interesting, especially because in practical cases RM structure usually evolves at a surface three-dimensionally from unintentional seed structure, but imaging diagnostics typically produce images that integrate along their line of sight. Therefore, uncharacterized, three-dimensional initial conditions preclude direct measurement of the RM features evolving within the mixing 
zone, in contrast with the present experiments.

The present experiments also offer the advantage of involving high Mach and Reynolds numbers, properties that are characteristic of many HED systems of interest. Shock tubes, the type of system on which much of existing experimental RM work has been performed, allow for the creation of very precise initial conditions and the use of high-resolution diagnostics $[16,17,18]$, but shock strength typically remains well below $M=2$. This low shock strength is also characteristic of the only previous experiments [19] to observe some elements of RM mode coupling. Previous studies employing lasers [20, 21] have involved single-mode interfaces, and these interfaces were also RT unstable, as their designs featured shocks encountering density drops. Further, when a shock is incident on such an interface, a rarefaction is reflected, and the growth subsequently occurs in an environment of varying density, meaning the growth parameters are constantly changing. Our experimental platform avoids these complications.

\section{Theoretical background}

If a material interface is seeded with a perturbation consisting of a single sinusoidal mode of initial amplitude $a^{\dagger}$ and wavenumber $k$ such that $k a^{\dagger} \ll 1$ and is then shocked, the perturbation amplitude $a$ will initially experience a phase of linear growth in time. Immediately when the shock encounters the interface, the interface experiences a short transient period, in which the perturbation is first compressed by the shock to an amplitude $a_{o}$, and then its growth rate increases until reaching a maximal, steady linear value $U_{\mathrm{RM}}[1,22]$.

This growth rate has been the subject of extensive previous study, owing to the difficulty in achieving a simple, reliable approach to its calculation. Rigorous linear treatment of the fluid equations, either by direct numerical solution [23] or by reformulating the fluid equations in terms of vorticity deposition $[24,25]$, produces equivalent results that generally capture the sensitivity of the growth rate to the fluid properties of the particular system being treated. However, implementation of either method requires sophisticated calculations. Simpler models typically start from the assumption that RM is driven by the impulsive acceleration of the interface by the passing shock $[1,22,26]$, but this assumption is not quite correct. Impulsive models produce tractable, closed-form results, but their predictions are only accurate under certain flow conditions, and it is not always immediately obvious a priori how the error depends upon the relevant parameters of the flow.

Allowing the system to evolve until $\mathrm{ka}$ exceeds about 0.1 causes it to enter a nonlinear growth phase [27, 28], in which the RM growth rate begins to decay, and eventually approaches zero following an asymptotic profile scaling as $(k t)^{-1}[29,30]$.

If the interface is seeded with two superposed sinusoidal modes $k_{1}$ and $k_{2}$, each of these will again initially grow linearly (and, consequently, independently) as just described. However, as the modes enter their nonlinear phase, they will couple and produce harmonics at wavenumbers $\left(k_{1} \pm k_{2}\right)$, at the expense of some of the growth of $k_{1}$ and $k_{2}$. Still later in time, the modes will begin to compete with each other, and due to the greater asymptotic velocity of the lower-frequency modes [19, 30], they will reabsorb the higher-frequency ones (so-called "bubble merging") on some characteristic time scale dependent upon their relative frequencies.

Finally, if the interface is seeded with many modes $\left\{k_{1}\right.$, $\left.k_{2}, \ldots, k_{i}\right\}$, it can be approximately treated as a spectrum. All of the above behavior will be observed and, in addition, the bubble-merging process will cause the characteristic wavelength $\langle\lambda\rangle$ of the interface structure to increase over time [31]. A property of such a system that has important ramifications for experimental design is the faster evolution of shorterwavelength modes than of longer-wavelength modes, since for a fixed duration of an experiment the shorter modes will evolve further. After sufficient time has passed, the rate of change of $\langle\lambda\rangle$ should asymptotically approach a power law of coefficient $\theta$, where $\theta$ has converged on some value. The higher-density RM spikes do not necessarily penetrate at the same rate as the lower-density bubbles do, so the overall interface is actually governed by the sum of two power laws. Theoretical predictions for a two-dimensional system estimate a value $\theta \sim 0.4$ for the bubbles, while the behavior of the spikes is sensitive to the Atwood number of the system. This value can range from around 0.4 , in the limit of low Atwood number, to 1 in the limit of high Atwood number [11, 12].

\section{Experimental conditions}

The experimental system is composed of four axially-stacked layers of material, shown in Fig. 1, with a cross-section width of $1100 \mu \mathrm{m}$ along the diagnostic line of sight. The first layer,

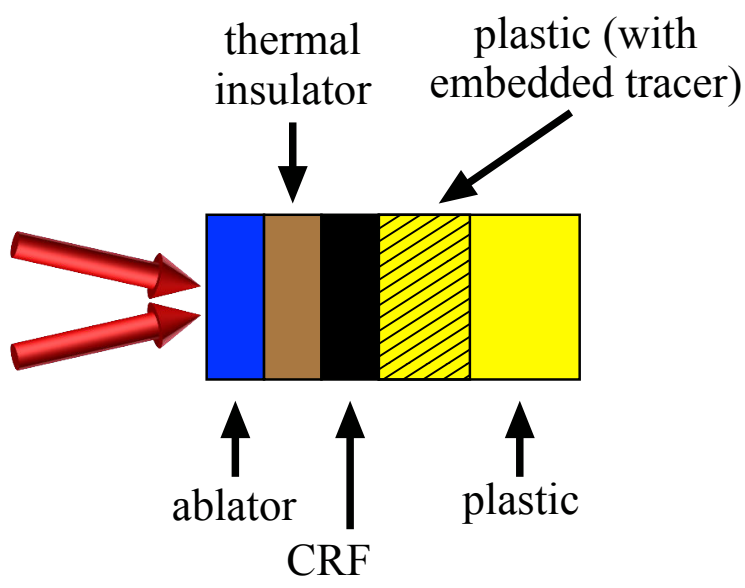

Figure 1: Schematic drawing of the experiment's physics assembly; red arrows indicate the direction of the laser drive. The orientation of the schematic is the same orientation of the radiographic data shown in Figs. 2.

a $125-\mu$ m-thick polycarbonate film (the ablator), is pressed against a second film functioning as a thermal insulator (TI), made of $150-\mu$ m-thick bromine-doped plastic $\left(\mathrm{C}_{50} \mathrm{H}_{48} \mathrm{Br}_{2}\right)$. Both layers are of density $1.2 \mathrm{~g} / \mathrm{cm}^{3}$. The purpose of the double layer is to allow the high- $Z$ bromine doping to prevent radiation and high-energy particles, generated by the laser's interaction with the plastic, from propagating through the system and heating the physics-relevant layers in advance of the shock's arrival, 
while avoiding direct irradiation of a layer including a high- $Z$ material, which would result in the emission of high-energy $X$ rays. Such $\mathrm{X}$ rays would both introduce unwanted signal onto the detector and heat the physics-relevant layers. Next, a 200$\mu \mathrm{m}$-thick layer of carbonized resorcinol formaldehyde (CRF, of density $0.4 \mathrm{~g} / \mathrm{cm}^{3}$ ) foam is pressed against the TI, and a block of polyamide-imide (PAI, $\mathrm{C}_{22} \mathrm{H}_{14} \mathrm{O}_{4} \mathrm{~N}_{2}$ ) plastic, of density 1.4 $\mathrm{g} / \mathrm{cm}^{3}$, is pressed against the CRF. This block features a 200$\mu$ m-wide tracer layer of iodine-doped plastic $\left(\mathrm{C}_{50} \mathrm{H}_{47} \mathrm{I}_{3}\right)$ at its center, extending $300 \mu \mathrm{m}$ down the system's axis. The higher- $Z$ iodine content of the tracer permits observation of the experiment at the center of the system, away from distorting boundary effects, by attenuating imaging $X$ rays at a much higher rate than occurs in the surrounding plastic. The interface between the CRF and the PAI block is where the RM growth is produced. A seed perturbation, with amplitude profile $a(y)$, where

$$
a(y)=5 \mu \mathrm{m} \cdot \cos \left(\frac{2 \pi y}{100 \mu \mathrm{m}}\right)-2.5 \mu \mathrm{m} \cdot \cos \left(\frac{2 \pi y}{50 \mu \mathrm{m}}\right),
$$

was machined into the face of the PAI block abutting the CRF, resulting in an overall profile with two modes. In one case, the block was machined flat to enable a baseline measurement of the interface. This design, with the shock passing from the lower-density CRF to the higher-density PAI block, enables the RM structure to grow within two uniformly shocked layers, avoiding phase reversal and growth of structure within a rarefaction. These last two properties were both present in the previous experiment of Glendinning et al. [20].

Alignment of the perturbation with respect to the diagnostic is critical to capturing the Fourier spectrum accurately, especially for shorter-wavelength modes. Depending on the direction of rotation, alignment error can potentially broaden the RM spikes, altering the mode content of the interface, and can reduce the amplitude. Therefore, we wish to characterize the accuracy of the alignment. The orientation of the perturbation with respect to the edge of the PAI block was confirmed to better than one tenth of a degree, and alignment of the block to the diagnostic was reproducible to within a few tenths of a degree. A typical rotational error, then, results in a misalignment of roughly one micron of the front of the tracer to its rear, as viewed by the diagnostic. This is small both with respect to the resolution limit of the diagnostic and with respect to the wavelengths we seek to observe.

Two ultraviolet OMEGA-EP [32] beams irradiate the ablator in sequence, driving a shock wave into the system, which then crosses the physics-relevant interface about 17 ns later. The beams have a $10 \mathrm{~ns}$, flat-topped temporal profile, with the tail end of the first pulse and the lead end of the second pulse tapered to allow the most stable profile possible during the transition, and a spatial profile of diameter $1100 \mu \mathrm{m}$ at full-width, half-maximum, smoothed by distributed phase plates. The intention of these parameters is that the combination of beams effectively behave as a single, long, flat-topped pulse. The first beam has an energy of approximately $4 \mathrm{~kJ}$, while the second has an energy of approximately $3 \mathrm{~kJ}$. These energies represent the maximum energies available at the time of the experiment. Because the purpose of the second beam is simply to sustain the ablation pressure on the target, preventing the already-launched shock from decaying into a blast wave, its lesser energy does not significantly affect the shock properties during the window of time in which the system is diagnosed. This property was extensively studied via simulations during the original design [13].

At some time following the beginning of the initial driving laser pulse, a third, infrared OMEGA-EP beam, with a 10-ps pulse duration and defocused to a spot size of diameter 200 $\mu \mathrm{m}$, irradiates a $20-\mu \mathrm{m}$-thick copper foil located $6 \mathrm{~mm}$ from the main physics target. This produces a flash of $\mathrm{X}$ rays at the copper $\mathrm{K}_{\alpha}$ energy of $8.0 \mathrm{keV}$. These $\mathrm{X}$ rays backlight the system, and are collected by a spherical-crystal imaging system (SCI) [33], producing a radiograph of the experiment on an imaging plate. Examples of these radiographs are shown in Fig. 2, for the two-mode initial perturbation described above, at $20 \mathrm{~ns}$ and $35 \mathrm{~ns}$. In these images, the ablator, $\mathrm{CRF}$, and plastic layers are
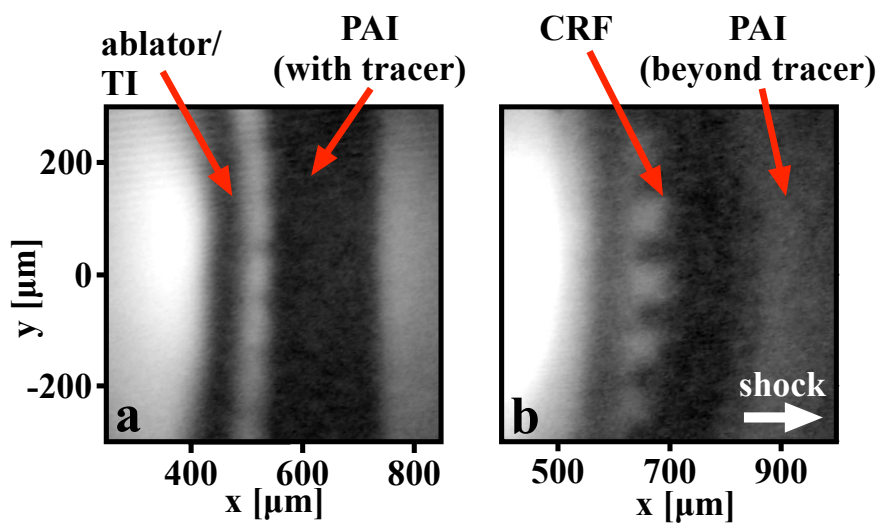

Figure 2: a. Experimental radiograph, taken at $20 \mathrm{~ns}$; b. Experimental radiograph, taken at $35 \mathrm{~ns}$. The RM growth of the interface structure during the elapsed time is evident from the two frames.

clearly identifiable. At $35 \mathrm{~ns}$, the shock has broken out of the tracer layer, and is identifiable in the undoped plastic layer. At $20 \mathrm{~ns}$, the shock can also be resolved, although the adjusted image contrast required for this would obscure the RM physics we show in the figure.

\section{One-dimensional flow}

The end goal of examining the one-dimensional hydrodynamics is to establish that the necessary conditions for observing clean, purely RM behavior have been achieved. This requires demonstrating that the shock and interface have steady velocities. In addition, by then simulating the system and reproducing the shock and interface motion correctly, we have confidence that we understand the hydrodynamic properties of the experiment, specifically the equation of state of the various material layers in the system and the strength of the initial driven shock.

Fig. 3 shows the measured locations, along with least-squares fits, of the shock and interface from the data. The trajectory of the shock supports a straight-line fit very well, indicating that we have driven a steady shock as we expected. From the 


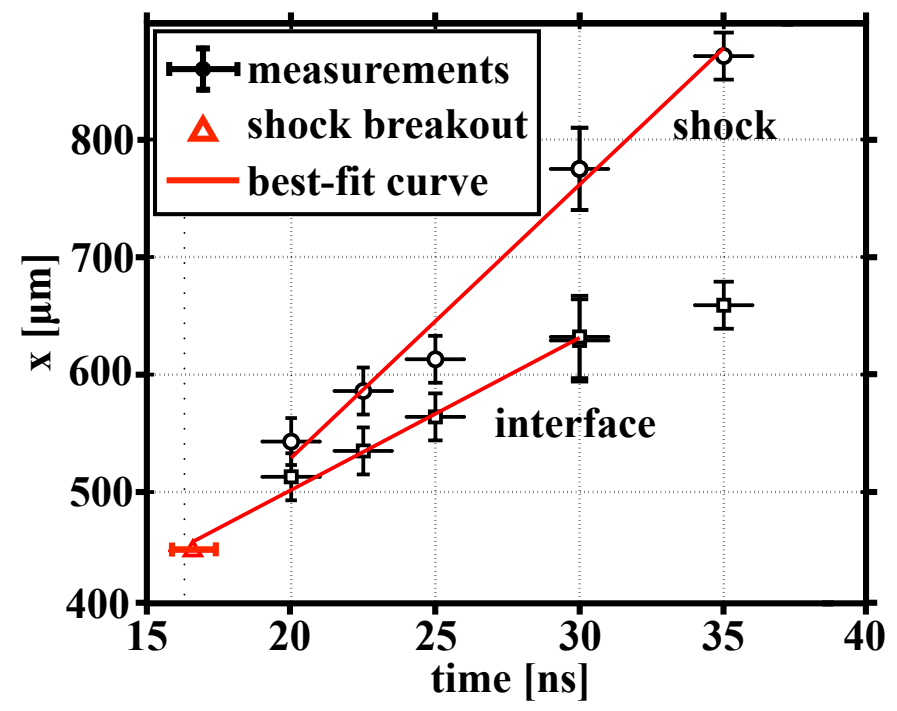

Figure 3: The trajectories of the shock and mean interface as measured in the data. Least-squares fits to the data are shown in red, and the extrapolated shock breakout time $t_{\mathrm{s}}$ is shown as a triangle. These quantities' values are listed in Table 1.

shock positions, we are able to extrapolate a breakout time, $t_{\mathrm{s}}$, shown as a triangle. Finally, the interface trajectory coupled with the shock breakout time also supports a straight-line fit well, providing further evidence that the one-dimensional flow is steady until at least $30 \mathrm{~ns}$.

The low displacement of the interface at $35 \mathrm{~ns}$, however, warrants closer inspection. Fig. 4 shows the same shock (black points) and interface (gray points) data overlaid upon a density map of the experiment in time, obtained from a onedimensional Hyades [34] simulation of the system. In this fig-

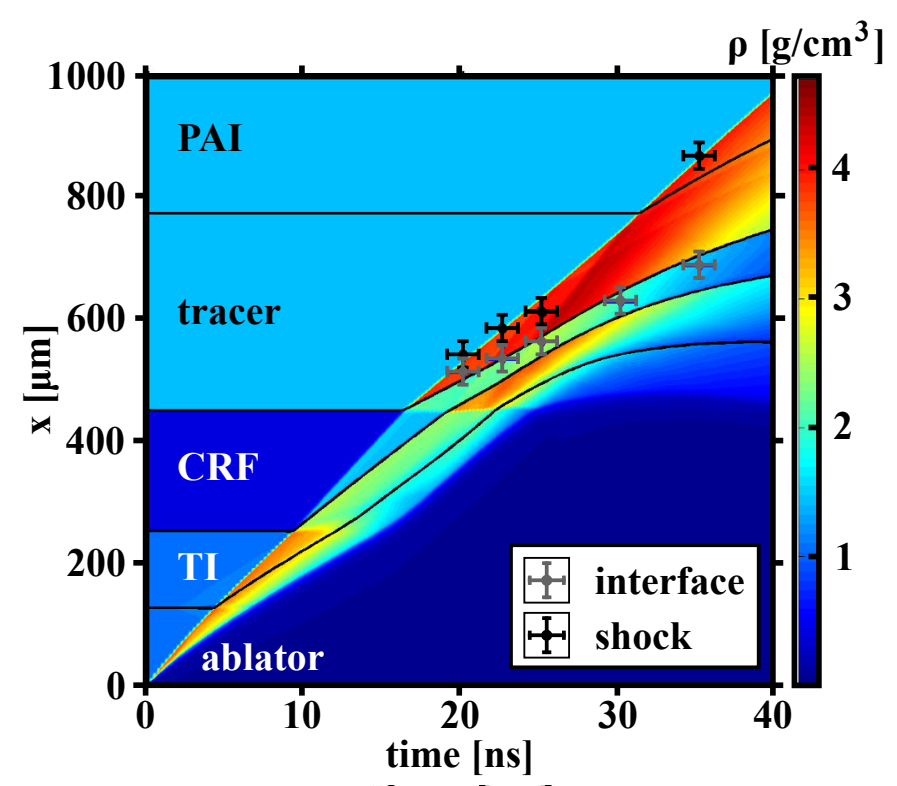

Figure 4: Density contour map showing the simulated one-dimensional evolution of the system. The measured locations of the shock and of the RM-relevant interface are overlaid as points, in black and grey respectively, for comparison. The positions of the various material interfaces are tracked by solid black lines.
Table 1: Properties of the one-dimensional flow, both of relevant velocities and of relevant interface parameters. Shown in the upper row are the incident and transmitted shock velocities $u_{\text {is }}$ and $u_{\text {ts }}$ as well as the shocked interface velocity $u_{\text {int }}$. Shown in the lower row are the shocked interface Atwood number $A_{\mathrm{PS}}$, the Mach number $M$, the pressure $P$, and the interface shock breakout time $t_{\mathrm{s}}$.

\begin{tabular}{lcccc}
\hline \hline & $\begin{array}{c}u_{\mathrm{is}} \\
{[\mu \mathrm{m} / \mathrm{ns}]}\end{array}$ & $\begin{array}{c}u_{\mathrm{ts}} \\
{[\mu \mathrm{m} / \mathrm{ns}]}\end{array}$ & $\begin{array}{c}u_{\mathrm{int}} \\
{[\mu \mathrm{m} / \mathrm{ns}]}\end{array}$ & \\
\hline $\begin{array}{l}\text { measured } \\
\text { simulated }\end{array}$ & 28 & $\begin{array}{c}23.2 \pm 1.6 \\
12.9 \pm 0.6\end{array}$ & \\
\hline \hline & $A_{\mathrm{PS}}$ & $M$ & $P[\mathrm{Mbar}]$ & $t_{\mathrm{s}}[\mathrm{ns}]$ \\
\hline measured & & & & $16.6 \pm 0.8$ \\
simulated & $1 / 3$ & 8 & 2.85 & $16.3 \pm 0.3$ \\
\hline
\end{tabular}

ure, the shock and the various material layers are clearly identified by the pronounced density jumps associated with them. Although the shock retains a steady velocity until $40 \mathrm{~ns}$, as evidenced by its straight trajectory in time, the interface velocity begins to fall off around $\sim 33 \mathrm{~ns}$. This indicates that, according to the simulation, the rarefaction associated with the disappearance of the ablation pressure as the driving laser pulse ends has propagated through the system and reached the interface around that time. Therefore, we expect that the interface location at $35 \mathrm{~ns}$ will not support a straight-line fit, and exclude it from the calculation. The RM growth is, in consequence, no longer occurring under strictly steady conditions beyond $~ 33$ $\mathrm{ns}$, although at $35 \mathrm{~ns}$ we expect the discrepancy to be small due to the short elapsed time since arrival of the rarefaction. In future experiments, we can readily extend this time limit by adding OMEGA-EP's fourth beam to the end of the driving laser pulse, lengthening the pulse by about $50 \%$ and adding several nanoseconds of steady interface motion [13].

Table 1 shows the RM-relevant properties of the onedimensional flow as measured in the experimental data where possible, as well as those and other relevant properties as predicted by the simulation. In this table, the Mach number $M$ is that of the incident shock, or the ratio of the incident shock velocity $u_{\text {is }}$ to the sound speed in the CRF, and the Atwood number $A_{\mathrm{PS}}$ is the ratio of the difference between the densities of the shocked plastic and CRF to their sum. The reported uncertainty of the quantities found by least-squares fits are the quantities' associated standard deviations, while the uncertainty of the simulated shock breakout time corresponds to an artificially-wide simulated shock front that takes time to traverse the interface. This artifact arises from the numerical methods used by the simulation and the consequent limited resolution of the shock front.

\section{Two-mode physics}

In order to extract the mode amplitudes from the data, the images were first processed by applying a Gaussian smoothing function over a small number of pixels, less than the resolution limit of the diagnostic, in order to mute small-scale noise, on the order of a few microns, in the images. The material interfaces, identified by steep intensity gradients in the images, were then sharpened using the unsharp-mask filtering technique [10, 35]. To do so, the image is first blurred using a two-dimensional 
Gaussian filter, and then the blurred image is subtracted from the original. Mathematically, the pixel-by-pixel operation to obtain the unsharp-mask image $I_{\mathrm{USM}}$ is expressed as

$$
I_{\mathrm{USM}}=I_{0}-I_{0} \otimes \frac{1}{\pi R^{2}} e^{-\frac{\left.\left(x-x_{0}\right)^{2}+\left(y-y_{0}\right)^{2}\right)}{R^{2}}} \Delta x \Delta y,
$$

where the second term on the right-hand side is the convolution of the matrix $I_{0}$ with a matrix having the Gaussian form on the right. In this equation, $I_{0}$ is the original image intensity value, $\Delta x$ and $\Delta y$ are the pixel dimensions (here, $2.5 \times 2.5$ $\mu \mathrm{m}), R$ is the Gaussian radius over which the filtering is applied, and $x_{0}$ and $y_{0}$ are the spatial coordinates of each given pixel. A value of $R=20 \mu \mathrm{m}$ produced the optimal result for our data. In the processed image, the contours of value zero then correspond to the locations of the material interfaces. An example of this process is shown in Fig. 5, for the 35-ns data from Fig. 2b. The radiograph is shown again in Fig. 5 a for reference,
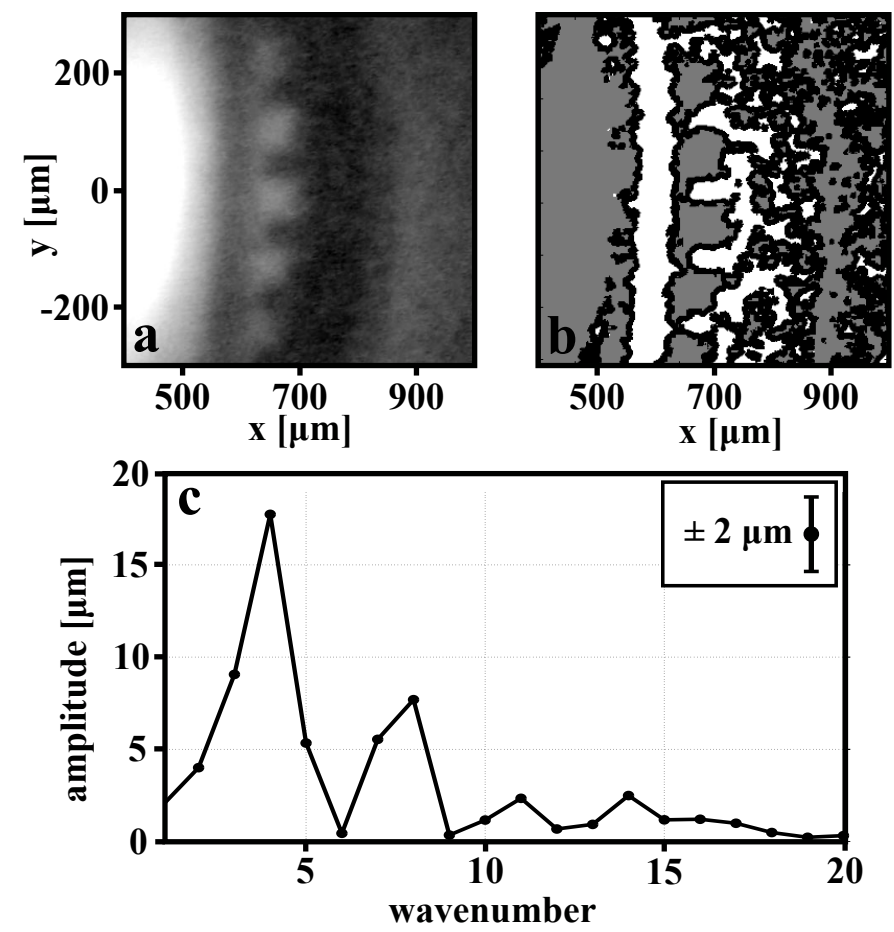

Figure 5: a. Experimental radiograph, taken at $35 \mathrm{~ns}$; b. Unsharp-mask filtering of the same radiograph, with the midway contour indicated by black lines; $c$. Fourier transform of the section of contour in Fig. 5b corresponding to the RM features, on the range $-200<y<200$.

while in Fig. 5b the zero contours are highlighted using black lines, and the color-mapping has been converted into a binary form; that is, all pixels where $I_{\mathrm{USM}}>0$ have an identical darker color, while all pixels where $I_{\mathrm{USM}}<0$ have an identical lighter color. The unsharp-mask process is most effective and accurate when the structures involved are thick compared to the pixel size, and when the overall intensity change is large. Thus, the unsharp mask picks out the CRF/ablator and RM-relevant interfaces well, and the shock somewhat less well. There is significant artifact present in areas of low intensity in the image, due to the greater relative magnitude of characteristic noise fluctuations in such locations.
The contour exhibiting RM growth can then be extracted for Fourier analysis. We begin by choosing the portion of the contour corresponding to the interface of interest. However, it is evident from Fig. $2 b$ that, in some places, noise in the image causes the contour to be multivalued in $x$. Therefore, we apply a binning algorithm to the image, such that at every pixel location in $y$ we retain only the leftmost value of $x$ from the interface contour.

We are then able to numerically apply a Fourier transform to the contour. Fig. 5c shows the result corresponding to Fig. 5b. Performing this technique on all data images allows us to track the time evolution both of the original, seeded interface modes, as well as of harmonic modes that have developed. The solid lines in Fig. 6 show the resulting growth of the three most prominent modes: the two original ones and their lowest coupled harmonic. (In this figure and elsewhere in the discussion

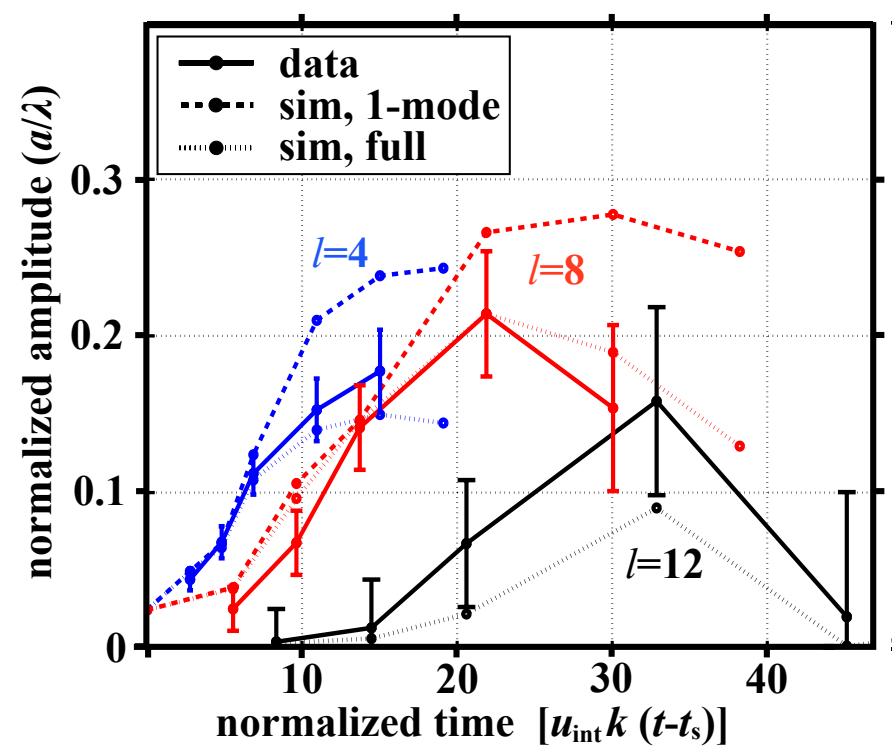

Figure 6: Time evolution of the two fundamental RM modes $(l=4,8)$ and their lowest coupled harmonic $(l=12)$, as measured using Fourier techniques. The solid lines correspond to the experimental radiographs, the finely-dotted lines to the full experimental simulation, and the heavily-dotted lines to the singlemode simulations. The five data points for each mode correspond to images taken at 20,22.5, 25, 30, and $35 \mathrm{~ns}$ in real time, respectively. Note that the time scale used is normalized to the standard, circular wavenumber $k$.

of the two-mode interface, we use the notation $l$ for the experimental mode wavenumbers, which are normalized such that $l=400 /(\lambda[\mu \mathrm{m}])$. This is to emphasize the contrast between our definition of $l$ and the standard notation for the non-normalized circular wavenumber $k=2 \pi / \lambda$. Thus, $l=4$ and $l=8$ correspond to the initial $\lambda=100 \mu \mathrm{m}$ and $\lambda=50 \mu \mathrm{m}$ modes, respectively, while $l=4+8=12$ corresponds to their coupled harmonic with $\lambda=33 \mu \mathrm{m}$.) The period of greatest growth for both original modes, corresponding to the time at which the modes have achieved their full linear growth rate, lasts until $a / \lambda$ is roughly $10-15 \%$. As the amplitude exceeds this magnitude, the nonlinear coupling effect begins to influence the modes' behavior. This is suggested by the appearance of the $l=12$ mode. To further confirm that mode coupling is responsible, rather than the natural, asymptotic saturation of the modes on their 
own, we performed two-dimensional simulations both of the full experimental system and of equivalent systems seeded with only one or the other of the original modes [10, 13]. The simulations were performed using the hydrodynamics code Dafna $[36,37]$, and initialized using the shock conditions produced by the Hyades simulation described in Section 4.

The results of the full and one-mode simulations are plotted in Fig. 6 as finely-dotted and heavily-dotted lines, respectively. These simulations clearly show that the growth rates begin to fall off earlier and at a smaller amplitude in each others' presence than occurs in isolation, and that the coupling effect is evident beginning at an amplitude-to-wavelength ratio of roughly $0.1-0.15$, as is expected from the theoretical discussion in Section 2. Further, at late time, the bubble-merger absorption of the shorter-wavelength modes by the longer-wavelength ones can also be observed. The agreement of the experimental result with the full simulation, combined with the our understanding of the one-dimensional hydrodynamics as described in Section 4 , give us confidence that the simulation provides a good prediction of the system's behavior.

As a further note regarding the simulated amplitudes in Fig. 6 , these were taken from simulation output that has been processed to mimic loss of resolution caused by the imaging diagnostic. As an example of how this resolution limitation can affect the measurements, we compare the amplitudes produced by the experiment, by the processed simulation, and by simulation without processing. These amplitudes, for the $l=4$, are plotted in Fig. 7. The processing was performed as follows.

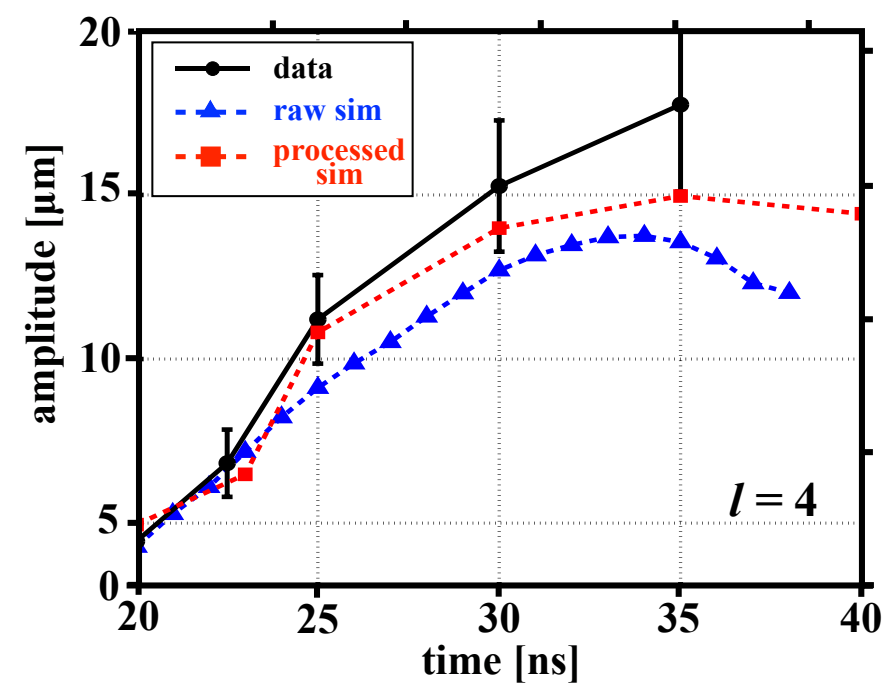

Figure 7: Comparison of the evolution of the $l=4$ mode according to the data (black with circles), the raw simulation output (blue with triangles), and the processed simulation output (red with squares).

First, the CRF and plastic areas in the 2D-simulation output are calibrated to the signal levels seen in the experimental radiograph. Next, we apply a moving-average smoothing filter with a $10 \mu \mathrm{m} \times 10 \mu \mathrm{m}$ area to the image, to simulate blurring due to the resolution limit imposed by the diagnostic (in our case, the limiting factor was the imaging plates) as well as blurring due to possible minor misalignment of the experimental system with respect to the diagnostic. Finally, we add multiplicative noise having a variance of the inverse of the signal-to-noise ratio (SNR) observed in the data, and then recover the contour using the same procedure described for the data. Signal issues are discussed in more detail in Appendix A.

In contrast, the amplitudes from the raw simulation were obtained from the exact shape of the interface given by the Dafna output. It can be seen in Fig. 7 that, in our scenario, the processing can introduce error of micron order into the Fourier spectrum, and we found that this error was fairly typical across the various modes and imaging times. Also, the behavior of the error was somewhat inconsistent from observation time to observation time at lower amplitudes. This gives us some insight into the error introduced by the process of making our observations, and we therefore treat the measured amplitudes with some caution.

Finally, we note one further complication in these spectral measurements, also captured by the simulations. This is the curvature of the shock front. Curvature arises because the driving laser spot is of finite size, and thus as the shock propagates it is also expanding outwards. The resulting curvature is then imparted on the interface as the shock passes. This effect can be clearly seen in all our data, including in those images shown in Fig. 2. Spectrally, this is the equivalent of introducing long-wavelength modes into the observed spectrum. These modes will also exhibit all the physical behavior we describe for the seeded modes in Section 2. Particularly, coupling between these modes and the $l=8$ mode is likely responsible for the slight decrease in amplitude of the simulated single $l=8$ mode at late times, since this behavior has no other ready physical explanation. This behavior is not observed in the simulated $l=4$ mode, probably because the smaller wavelength difference between the $l=4$ mode and the same curvature-induced modes results in a slower time scale for the effect to appear.

\section{Conclusions}

We have presented experiments which permit the direct observation of nonlinear RM structure, under strong and steady shock conditions, from well-characterized initial conditions. Results from a two-mode system demonstrate the onset of the nonlinear phenomena of mode coupling and subsequent mode reabsorption, or bubble merger. This system also serves as a test case for our data processing and Fourier analysis technique. Our results with this technique applied to the two-mode experiment show that we can cleanly produce and measure the physics without depending upon direct visual identification of the features in question. A particular future goal of these experiments is to use a carefully-chosen complex initial perturbation to push the system to self-similarity, in a manner that could be measured clearly from the Fourier spectrum at the interface $[13,38]$.

\section{Appendix A. Diagnostic considerations}

The introduction of the long drive $(\sim 20 \mathrm{~ns})$ into this experiment poses several diagnostic challenges, making it difficult to 
employ traditional imaging techniques such as point-projection backlighting. First of all, the extended length of the driving pulse causes secondary emission to occur at the expanding ablation front, which will saturate the detector absent some manner of shielding. Second, the sustained ablation pressure due to the long drive pulse prevents the system from rarefying in the axial direction, which causes the materials at the RM-relevant interface to remain compressed at the imaging time. Finally, the CRF density of $0.4 \mathrm{~g} / \mathrm{cm}^{3}$ needed to create the desired physics is already high compared to that present in many other similar experiments, which have typically used CRF of density of around $0.1 \mathrm{~g} / \mathrm{cm}^{3}[9,39,40]$.

Typically, the first issue can be solved by using a gated diagnostic, such as the X-ray Framing Camera at the OMEGAEP facility. However, because of the densities involved, use of a backlighting material of high-enough $Z$ (and hence of highenough X-ray energy) to achieve acceptable transmission fraction through the CRF results in an X-ray conversion efficiency too low to achieve sufficient contrast. An attempt to image this experiment using this diagnostic resulted in a signal-to-noise ratio of roughly 0.7 . The noise structure was on the order of several microns in size, and of an amplitude of roughly the contrast between the CRF and plastic.

The crystal-imaging diagnostic, data examples of which were shown in Fig. 2, addresses all of these diagnostic issues through the use of a different method of creating the X-ray beam. First, rather than employing a pinhole, which blocks the majority of the photons in order to create the imaging beam, the curved crystal focuses them directly, and the only limit on the useful signal is the collection area of the crystal itself. Second, because of the geometry of the crystal's imaging path, it is possible to shield the detector in such a way that nearly all unwanted emission is prevented from interfering with the diagnostic [33].

\section{Acknowledgments}

The authors would like to thank Robb Gillespie, of the University of Michigan, and General Atomics, who between them fabricated most of the parts used in these experiments. We would also like to thank Chad Mileham and Christian Stoeckl for productive conversations about the SCI diagnostic, as well as Yoni Elbaz both for productive conversations about the RM physics and for authorization to use the Dafna code in our work presented here. Lawrence Livermore National Laboratory provided the foams and doped plastics used in the experiments.

This work is funded by the NNSA-DS and SC-OFES Joint Program in High-Energy-Density Laboratory Plasmas, grant number DE-NA0001840, and by the National Laser User Facility Program, grant number DE-NA0002032, and through the Laboratory for Laser Energetics, University of Rochester by the NNSA/OICF under Cooperative Agreement No. DE-FC5208NA28302.

[1] R. D. Richtmyer, Taylor instability in shock acceleration of compressible fluids, Communications on Pure and Applied Mathematics 13 (1960) 297-319.

[2] E. E. Meshkov, Instability of the interface of two gases accelerated by a shock wave, Soviet Fluid Dynamics 4 (1969) 101-104.
[3] Y. Aglitskiy, A. L. Velikovich, M. Karasik, N. Metzler, S. T. Zalesak, A. J. Schmitt, L. Phillips, J. H. Gardner, V. Serlin, J. L. Weaver, S. P. Obenschain, Basic hydrodynamics of Richtmyer-Meshkov-type growth and oscillations in the inertial confinement fusion-relevant conditions, Philosophical Transactions of the Royal Society A: Mathematical, Physical and Engineering Sciences 368 (2010) 1739-1768.

[4] J. Matsumoto, Y. Masada, Rayleigh-Taylor and Richtmyer-Meshkov instabilities in relativistic hydrodynamic jets, in: European Physical Journal Web of Conferences, volume 61 of European Physical Journal Web of Conferences, p. 2005.

[5] J. Kane, R. P. Drake, B. A. Remington, An evaluation of the RichtmyerMeshkov instability in supernova remnant formation, The Astrophysical Journal 511 (1999) 335-340.

[6] L. Rayleigh, Scientific Papers, volume II, Cambridge Univ. Press, Cambridge, England, 1900.

[7] G. Taylor, The instability of liquid surfaces when accelerated in a direction perpendicular to their planes. I, Royal Society of London Proceedings Series A 201 (1950) 192-196.

[8] R. A. Chevalier, The hydrodynamics of Type II supernovae, The Astrophysical Journal 207 (1976) 872-887.

[9] H. F. Robey, J. O. Kane, B. A. Remington, R. P. Drake, O. A. Hurricane, H. Louis, R. J. Wallace, J. Knauer, P. A. Keiter, D. Arnett, D. D. Ryutov, An experimental testbed for the study of hydrodynamic issues in supernovae, Physics of Plasmas 8 (2001) 2446-2453.

[10] C. A. Di Stefano, G. Malamud, C. C. Kuranz, S. R. Klein, C. Stoeckl, R. P. Drake, Richtmyer-Meshkov evolution under steady shock conditions in the high-energy-density regime, Applied Physics Letters 106 (2015).

[11] U. Alon, J. Hecht, D. Mukamel, D. Shvarts, Scale invariant mixing rates of hydrodynamically unstable interfaces, Phys. Rev. Lett. 72 (1994) 2867-2870.

[12] U. Alon, J. Hecht, D. Ofer, D. Shvarts, Power laws and similarity of Rayleigh-Taylor and Richtmyer-Meshkov mixing fronts at all density ratios, Phys. Rev. Lett. 74 (1995) 534-537.

[13] G. Malamud, C. A. Di Stefano, Y. Elbaz, C. M. Huntington, C. C. Kuranz, P. A. Keiter, R. P. Drake, A design of a two-dimensional, multimode RM experiment on OMEGA-EP, High Energy Density Physics 9 (2013) 122 $-131$.

[14] G. Dimonte, Nonlinear evolution of the Rayleigh-Taylor and RichtmyerMeshkov instabilities, Physics of Plasmas 6 (1999) 2009-2015.

[15] D. Oron, L. Arazi, D. Kartoon, A. Rikanati, U. Alon, D. Shvarts, Dimensionality dependence of the Rayleigh-Taylor and Richtmyer-Meshkov instability late-time scaling laws, Physics of Plasmas 8 (2001) 28832889.

[16] M. A. Jones, J. W. Jacobs, A membraneless experiment for the study of Richtmyer-Meshkov instability of a shock-accelerated gas interface, Physics of Fluids 9 (1997) 3078-3085.

[17] B. D. Collins, J. W. Jacobs, PLIF flow visualization and measurements of the Richtmyer-Meshkov instability of an air/SF6 interface, Journal of Fluid Mechanics 464 (2002) 113-136.

[18] C. C. Long, V. V. Krivets, J. A. Greenough, J. W. Jacobs, Shock tube experiments and numerical simulation of the single-mode, threedimensional Richtmyer-Meshkov instability, Physics of Fluids (1994present) 21 (2009).

[19] O. Sadot, L. Erez, U. Alon, D. Oron, L. A. Levin, G. Erez, G. Ben-Dor, D. Shvarts, Study of nonlinear evolution of single-mode and two-bubble interaction under Richtmyer-Meshkov instability, Phys. Rev. Lett. 80 (1998) 1654-1657.

[20] S. G. Glendinning, J. Bolstad, D. G. Braun, M. J. Edwards, W. W. Hsing, B. F. Lasinski, H. Louis, A. Miles, J. Moreno, T. A. Peyser, B. A. Remington, H. F. Robey, E. J. Turano, C. P. Verdon, Y. Zhou, Effect of shock proximity on Richtmyer-Meshkov growth, Physics of Plasmas 10 (2003) 1931-1936.

[21] B. A. Remington, S. V. Weber, M. M. Marinak, S. W. Haan, J. D. Kilkenny, R. Wallace, G. Dimonte, Multimode Rayleigh-Taylor experiments on Nova, Phys. Rev. Lett. 73 (1994) 545-548.

[22] K. A. Meyer, P. J. Blewett, Numerical investigation of the stability of a shock accelerated interface between two fluids, Physics of Fluids (19581988) 15 (1972) 753-759.

[23] Y. Yang, Q. Zhang, D. H. Sharp, Small amplitude theory of RichtmyerMeshkov instability, Physics of Fluids 6 (1994) 1856-1873.

[24] J. G. Wouchuk, Growth rate of the linear Richtmyer-Meshkov instability 
when a shock is reflected, Phys. Rev. E 63 (2001) 056303.

[25] J. G. Wouchuk, K. Nishihara, Asymptotic growth in the linear Richtmyer-Meshkov instability, Physics of Plasmas 4 (1997) 1028-1038.

[26] M. Vandenboomgaerde, C. Mügler, S. Gauthier, Impulsive model for the Richtmyer-Meshkov instability, Phys. Rev. E 58 (1998) 1874-1882.

[27] Q. Zhang, S.-I. Sohn, An analytical nonlinear theory of RichtmyerMeshkov instability, Physics Letters A 212 (1996) $149-155$.

[28] Q. Zhang, S.-I. Sohn, Nonlinear theory of unstable fluid mixing driven by shock wave, Physics of Fluids 9 (1997) 1106-1124.

[29] S. W. Haan, S. M. Pollaine, J. D. Lindl, L. J. Suter, R. L. Berger, L. V. Powers, W. E. Alley, P. A. Amendt, J. A. Futterman, W. K. Levedahl, M. D. Rosen, D. P. Rowley, R. A. Sacks, A. I. Shestakov, G. L. Strobel, M. Tabak, S. V. Weber, G. B. Zimmerman, W. J. Krauser, D. C. Wilson, S. V. Coggeshall, D. B. Harris, N. M. Hoffman, B. H. Wilde, Design and modeling of ignition targets for the National Ignition Facility, Physics of Plasmas 2 (1995) 2480-2487.

[30] J. Hecht, U. Alon, D. Shvarts, Potential flow models of Rayleigh-Taylor and Richtmyer-Meshkov bubble fronts, Physics of Fluids 6 (1994) 4019 4030.

[31] D. Ofer, U. Alon, D. Shvarts, R. L. McCrory, C. P. Verdon, Modal model for the nonlinear multimode Rayleigh-Taylor instability, Physics of Plasmas 3 (1996) 3073-3090.

[32] D. N. Maywar, J. H. Kelly, L. J. Waxer, S. F. B. Morse, I. A. Begishev, J. Bromage, C. Dorrer, J. L. Edwards, L. Folnsbee, M. J. Guardalben, S. D. Jacobs, R. Jungquist, T. J. Kessler, R. W. Kidder, B. E. Kruschwitz, S. J. Loucks, J. R. Marciante, R. L. McCrory, D. D. Meyerhofer, A. V. Okishev, J. B. Oliver, G. Pien, J. Qiao, J. Puth, A. L. Rigatti, A. W. Schmid, M. J. S. III, C. Stoeckl, K. A. Thorp, J. D. Zuegel, OMEGA EP highenergy petawatt laser: progress and prospects, Journal of Physics: Conference Series 112 (2008) 032007.

[33] C. Stoeckl, G. Fiksel, D. Guy, C. Mileham, P. M. Nilson, T. C. Sangster, M. J. Shoup, 3rd, W. Theobald, A spherical crystal imager for OMEGA EP, Rev Sci Instrum 83 (2012) 033107.

[34] J. T. Larsen, S. M. Lane, HYADES: a plasma hydrodynamics code for dense plasma studies, Journal of Quantitative Spectroscopy and Radiative Transfer 51 (1994) $179-186$.

[35] G. Malamud, A. Shimony, W. C. Wan, C. A. Di Stefano, Y. Elbaz, C. C. Kuranz, P. A. Keiter, R. P. Drake, D. Shvarts, A design of a twodimensional, supersonic KH experiment on OMEGA-EP, High Energy Density Physics 9 (2013) 672-686.

[36] A. M. Khokhlov, Fully threaded tree algorithms for adaptive refinement fluid dynamics simulations, Journal of Computational Physics 143 (1998) 519-543.

[37] G. H. Miller, E. G. Puckett, A high-order Godunov method for multiple condensed phases, Journal of Computational Physics 128 (1996) 134 164.

[38] B. Thornber, D. Drikakis, D. L. Youngs, R. J. R. Williams, The influence of initial conditions on turbulent mixing due to Richtmyer-Meshkov instability, Journal of Fluid Mechanics 654 (2010) 99-139.

[39] G. Dimonte, B. A. Remington, Richtmyer-Meshkov experiments on the Nova laser at high compression, Phys. Rev. Lett. 70 (1993) 1806-1809.

[40] E. C. Harding, J. F. Hansen, O. A. Hurricane, R. P. Drake, H. F. Robey, C. C. Kuranz, B. A. Remington, M. J. Bono, M. J. Grosskopf, R. S. Gillespie, Observation of a Kelvin-Helmholtz instability in a high-energydensity plasma on the Omega laser, Physical Review Letters 103 (2009) 045005 